\title{
INTERAÇÕES ENTRE FEROMÔNIOS DE INSETOS E SEMIOQUÍMICOS DE PLANTAS
}

\author{
Insect Pheromones and Plant Semiochemicals Interactions
}

Fabiane Cristina Ceruti

Bióloga, Doutoranda em Entomologia - UFPR. E-mail: biaceruti@ufpr.br.

\begin{abstract}
Resumo
Os semioquímicos de plantas são conhecidos por produzir uma extensa gama de respostas no comportamento dos insetos. Alguns insetos seqüestram ou adquirem compostos da planta hospedeira e os usam como feromônios sexuais ou seus precursores. Outros insetos produzem ou liberam feromônios sexuais em resposta à presença da sua planta hospedeira específica. Os compostos químicos da planta hospedeira muitas vezes sinergizam a resposta do inseto ao feromônio sexual. Os voláteis da planta também podem ter efeito inibitório ou repelente que interrompem as respostas dos insetos aos feromônios e atraem predadores e parasitóides para atacar as espécies após o dano de herbivoria. Esta revisão descreve diferentes interações entre semioquímicos de plantas e feromônios de insetos, enfatizando aquelas que podem resultar no desenvolvimento de programas mais eficientes e seguros para controle de pragas.
\end{abstract}

Palavras-chave: Comportamento de insetos; Voláteis de plantas; Controle de pragas.

\begin{abstract}
The plant semiochemicals are known to produce a wide range of behavioral responses in insects. Some insects sequester or acquire host plant compounds and use them as sex pheromones or its precursors. Other insects produce or release sex pheromones in response to its specific host plant. Chemical compounds from host plants often synergistically enhance the response of an insect to sex pheromones. The plant volatiles can also have inhibitory or repellent effects that interrupt insect responses to pheromones and attract predators and parasitoids to the attacking species after herbivory injury. This review describes different interactions between plant semiochemicals and insect pheromones, emphasizing in those wich can result in the development of more efficient and reliable programs for pest control.
\end{abstract}

Keywords: Insects behavior; Plant volatiles; Pest control. 


\section{INTRODUÇÃO}

A comunicação é parte integrante do comportamento animal, que é definida como um processo que envolve a transmissão de sinais entre organismos. Em algumas situações, confere vantagens apenas para o organismo emissor e seu grupo; em outras, apenas para os organismos receptores ou para ambos (NASCIMENTO et al., 2001).

Os insetos são os seres vivos que mais utilizam os odores para desempenhar suas funções vitais (localização de presas, defesa e agressividade, seleção de plantas hospedeiras, escolha dos locais para oviposição, corte e acasalamento) (TEGONI et al., 2004). Essa comunicação pode ser efetuada entre organismos da mesma espécie (feromônios - substâncias secretadas por um indivíduo e recebidas por um segundo, provocando uma reação específica ou um processo de desenvolvimento fisiológico específico) ou entre espécies diferentes (cairomônios, alomônios, sinomônios).

Vários estudos realizados com feromônios de insetos têm reconhecido que essas substâncias são a chave para o sistema de comunicação intra-específica. Em estudos mais recentes sobre as interações entre feromônios de insetos e semioquímicos (moléculas que levam sinais de um organismo para outro) da planta hospedeira apresentaram resultados importantes para a compreensão de algumas interações envolvendo a comunicação interespecífica. Tais interações são manifestadas como efeito das plantas hospedeiras na fisiologia e comportamento dos insetos, refletindo diferentes tipos de estratégias para aperfeiçoar a alimentação, acasalamento e reprodução (LANDOLT; PHILLIPS, 1997).

As plantas hospedeiras têm um papel importante na origem do feromônio sexual de alguns insetos fitófagos, por meio da aquisição de químicos bioativos e requerem precursores químicos de feromônio via consumo, absorção ou inalação de material da planta hospedeira (REDDY; GUERRERO, 2004).

Alguns insetos adquirem compostos químicos da planta hospedeira e os usam como feromônios sexuais ou precursores de feromônio. Os primeiros estudos com identificação de feromônios das espécies de besouros-da-casca (Coleoptera: Scolytidae), cuja biologia do feromônio sexual, incluindo a biossíntese de feromônio e a atratividade, são integrados com os odores químicos da árvore hospedeira, foram realizados por Wood (1982). A mediação do feromônio na agregação dos coleópteros, particularmente aqueles atrativos produzidos pelos machos, é reconhecido por estar inter-relacionados com a presença de alimento (material da planta hospedeira) nos trabalhos de Borden (2003).

Os voláteis da planta hospedeira podem induzir a produção ou liberação de feromônios em certos insetos e também podem ter um efeito inibitório ou repelente, interrompendo a resposta dos insetos aos seus próprios feromônios. Em outros casos, compostos derivados da planta hospedeira, após um ataque de herbívoro, podem atrair predadores, servindo como um mecanismo de defesa para a planta (REDDY; GUERRERO, 2004).

O papel das plantas hospedeiras na comunicação sexual das mariposas foi revisado por McNeil e Delisle (1989). Em várias revisões sobre os feromônios de besouros-da-casca, o comportamento de agregação e as relações com a planta hospedeira envolvem discussões dos efeitos da planta na comunicação pelo feromônio. Eisner e Meinwald (1997) realizaram uma revisão dos alcalóides da planta hospedeira como precursores de feromônios de certas borboletas e mariposas.

O objetivo desta revisão é identificar quais os tipos de interações e influências que têm sido observadas entre os semioquímicos das plantas hospedeiras e os feromônios dos insetos. Os objetivos específicos são:

(a) Descrever como estas influências ocorrem em termos do comportamento do inseto (mecanismo comportamental).

(b) Discutir prováveis estratégias dos insetos que explicam as respostas ao estímulo da planta para aperfeiçoar a reprodução.

(c) Descrever algumas interações que possam contribuir para o desenvolvimento de programas de manejo de pragas. 


\section{Estímulo da planta hospedeira na produção de feromônios em insetos}

As plantas hospedeiras exercem um papel fundamental na produção e uso de feromônios sexuais por insetos herbívoros, por meio do seqüestro (aquisição) de compostos químicos ativos e precursores de feromônios sexuais da planta hospedeira, na forma jovem ou adulta do inseto (NISHIDA, 2002). Um dos melhores exemplos do papel da planta hospedeira na produção de feromônio sexual é o seqüestro de compostos químicos da planta hospedeira pelas larvas e seu subseqüente uso na comunicação sexual dos adultos. Possivelmente, o primeiro trabalho sobre compostos químicos de plantas hospedeiras seqüestrados por larvas e utilizados como feromônio sexual de adultos foi o estudo com Archips semiferana (Lepidoptera: Tortricidae) (HENDRY et al., 1975). Estudos em vários gêneros de Arctiidae e certos grupos de Nymphalidae demonstraram que os compostos secundários das plantas consumidos pelas larvas são usados pelos machos adultos na atração sexual ou interações de corte (HENDRY, 1976).

Os machos de Utetheisa ornatrix (Lepidoptera: Arctiidae) possuem um feromônio de corte, hidroxidanaidal, que é derivado de alcalóides pirrolizidínicos ingeridos no estágio larval das plantas hospedeiras Crotalaria spectabilis (Fabaceae) (CONNER et al., 1990; DUSSOURD et al., 1988). As larvas de $U$. ornatrix seqüestram alcalóides pirrolizidínicos (monocrotalina) e os retêm durante os estágios de desenvolvimento. Quando adulto, o derivado alcalóide é transferido para a fêmea durante a cópula (via espermatóforo) e na oviposição ela repassa os alcalóides com os ovos, servindo como proteção (GONZÁLEZ et al., 1999; IYENGAR et al., 2001).

Exemplos de alcalóides pirrolizidínicos seqüestrados por larvas e ninfas são encontrados em espécies de Lepidoptera, Coleoptera, Orthoptera e Hemiptera (certos afídeos). Esses alcalóides são hepatotóxicos e pneumotóxicos para os vertebrados e genotóxicos para os insetos (HARTMANN, 1999). Nos insetos, eles são armazenados e mantidos na forma de óxidos não tóxicos e quando se alimentam, eles são modificados no intestino e adsorvidos como alcalóides terciários (HARTMANN, 1999). Os insetos podem utilizar esses alcalóides como defesa química (alimento deterrente ou coloração aposemática) contra invertebrados predadores, como as aranhas, formigas, coccinelídeos e vespas (NISHIDA, 2002). Os machos advertem sua carga de alcalóides pela sinalização com hidroxidanaidal, fornecendo às fêmeas uma maneira de discriminar entre machos que possuem o alcalóide e machos sem eles.

Os machos adultos também podem obter alcalóides pirrolizidínicos das plantas e usá-los como precursores de feromônios. Isso ocorre em alguns Arctiidae e na maior parte das borboletas Danainae e Ithomiinae. Como exemplo, os machos de Cisseps fulvicollis (Lepidoptera: Arctiidae), obtêm alcalóides pirrolizidínicos das plantas e os usam como precursores para produção de feromônio sexual. Nessa espécie, os adultos adquirem alcalóides pirrolizidínicos com o néctar ou, mais freqüentemente, quando se alimentam de partes da planta danificadas ou mortas que contêm estes compostos (HARTMANN; OBER, 2000). Alguns adultos de Danaiinae também se alimentam de plantas contendo alcalóides e os usam como precursores bioquímicos de feromônios de corte usados para atrair as fêmeas em aproximação (SCHNEIDER et al., 1975; BOPPRE, 1978). BOPPRE, (1978) descreveu a seqüência de corte e acasalamento da borboleta Danaus gillipus (Lepidoptera: Nymphalidae), na qual o macho inicia o processo de corte por meio de sinais visuais. Ele sobrevoa a fêmea enquanto expõe constantemente o tufo de pêlos. Desta estrutura, são liberados alcalóides pirrolizidínicos que estimulam a receptividade da fêmea, desencadeando o processo de cópula.

A produção de feromônio sexual pelas fêmeas de Helicoverpa zea (Lepidoptera: Noctuidae), uma praga do milho, é induzida pela presença da planta hospedeira (RAINA et al., 1992). Os voláteis de compostos químicos do milho, extraídos com solvente do extrato desse, mostram a produção do feromônio sexual nas glândulas terminais do abdômen de H. zea. As mariposas removidas da planta hospedeira não tiveram atividade reprodutiva, incluindo a produção de feromônio sexual. Um exemplo similar foi demonstrado com Helicoverpa phloxiphaga (Lepidoptera: Noctuidae), a qual necessitou da presença da planta hospedeira Castilleja indivisa (Scrophulariaceae) para a produção do feromônio sexual (RAINA, 1988).

A aquisição ou coleta de compostos químicos das plantas e seu uso com função sexual é também conhecido para certas espécies de abelhas Euglossinae (DRESSLER, 1982; WILLIAMS, 2002). Os 
machos das abelhas coletam compostos químicos das orquídeas, raspando a camada de cera da cutícula das plantas e armazenando o material na corbícula das pernas coletoras. O material, uma mistura de compostos terpenóides, é usado como feromônio de agregação para promover a formação de leks (lugar onde os machos competem pelas fêmeas) ou como atrativo sexual.

Muitas espécies de coleópteros, principalmente escolitídeos (besouros-da-casca), alimentamse e agregam-se para acasalar na planta hospedeira, como um resultado da atração pelo feromônio sexual, e a síntese do feromônio nos machos é estimulada pela alimentação na árvore hospedeira (WOOD, 1982; BIRCH, 1994; BORDEN, 2003). Certas espécies de escolitídeos são conhecidas por produzir feromônios terpenóides a partir dos terpenos da planta hospedeira. Os machos de Ips paraconfusus usam o mirceno das plantas hospedeiras para produzir os feromônios atrativos ipsenol e ipsdienol, que são liberados após formação de galeria na planta hospedeira (BYERS, 1982). Para os machos de I. paraconfusus e Ips avulsus, a exposição aos vapores provenientes do mirceno são suficientes para a produção do feromônio, mas os machos de Ips calligraphus e Ips grandicollis precisam alimentar-se antes para o mirceno ser convertido em ipsdienol (BYERS, 1982). Outras espécies podem seqüestrar mirceno enquanto larvas na adição para aquisição de mirceno na alimentação quando adultos (RAFFA et al., 1996). Os machos de Dendroctonus ponderosae (Coleoptera: Scolytidae) usam a-pineno das árvores hospedeiras como um precursor para produção de feromônio trans-verbenol (HUGHES, 1973). Assim, a exposição ao mirceno volátil aumenta a quantidade de ipsenol e ipsdienol nos tecidos do intestino posterior dos machos de I. paraconfusus (BYERS, 1982) e de outros Dendroctonus spp. (HUNT et al., 1986). Essa afirmação foi comprovada em estudos de fisiologia, pela aplicação de $\mathrm{H}^{2}$-mirceno e a-pineno marcados radioativamente nos insetos, resultando na produção de $\mathrm{H}^{2}$-ipsenol, $\mathrm{H}^{2}$-ipsdienol e cis- e trans-verbenol (BARKAWI et al., 2003).

\section{Estímulo da planta hospedeira na liberação de feromônios em insetos}

O estímulo para liberação de feromônio pelos voláteis de plantas hospedeiras ocorre em muitas espécies de Coleoptera e Lepidoptera. Nos Coleoptera, algumas espécies, incluindo o bicudo-doalgodoeiro, Anthonomus grandis (Curculionidae), liberam feromônio após a alimentação na planta hospedeira, mas isso tem sido comprovado em poucos casos (DICKENS, 1989). Contudo, foi verificado que a palmeira, Elaeis quineensis (Arecaceae), uma planta hospedeira de Rhyncophorus phoenicis (Curculionidae), produz uma mistura de ésteres voláteis, onde o etil acetato induz os machos a liberarem o feromônio (E)-6-metil-2-hepten-4-ol (rincoforol) (JAFFE et al., 1993). Esse composto não é ativo no campo a menos que misturado com etil acetato ou outros voláteis da planta hospedeira como hexanal, isoamil acetato ou isopentanol. Em vista desses resultados, armadilhas com rincoforol, cana-de-açúcar e etil acetato têm sido recomendadas para o controle de infestações por $R$. phoenicis (JAFFE et al., 1993; ROCHAT et al., 2000).

Nos Lepidoptera existem poucos estudos com a indução dos voláteis de plantas na liberação de feromônio, provavelmente porque as mariposas facilmente distribuem moléculas de feromônio, até mesmo na ausência de voláteis de plantas hospedeiras. Verificou-se que a mariposa Antherea polyphemus (Saturniidae) adquire parte das folhagens da planta hospedeira, o carvalho vermelho Quercus rubra (Ulmaceae), para estimular o acasalamento devido ao composto químico trans-2-hexanal da planta favorecer a liberação de feromônio sexual pelas fêmeas (RIDDIFORD; WILLIAMS, 1997). As fêmeas da mariposa do girassol, Homeosoma electellum (Pyralidae) são estimuladas para liberar o feromônio sexual pela presença do pólen da planta hospedeira (MCNEIL; DELISLE, 1989). As fêmeas de quatro espécies de Yponomeuta (Yponomeutidae) são estimuladas pelas suas plantas hospedeiras para liberação do feromônio sexual atrativo para os machos (HENDRIKSE; VON-BUNNEMYER, 1987). Nessas espécies, as fêmeas são atraídas primeiramente para suas plantas hospedeiras, para então liberarem o feromônio sexual.

\section{Efeito repelente induzido pelos semioquímicos}

A localização da planta hospedeira é freqüentemente resultado de um sinal químico e/ou visual. Isso implica que os insetos são capazes de detectar a planta hospedeira enquanto voam e também 
que o fator de seleção do hospedeiro pode ser dependente da falta de repelência. Compostos da planta hospedeira podem ter um efeito inibitório ou repelente em adição a seus atrativos primários, precursores e sinergistas de feromônios. Contudo, em contraste com os estudos bem documentados da natureza atrativa ou sinergista de muitos compostos químicos, o efeito repelente ou inibitório de outros semioquímicos da planta hospedeira merecem estudos para investigação. Um exemplo representativo é 4-alil anisole, um composto comum produzido por Pinus taeda (Pinaceae) e algumas espécies de coníferas, que significativamente reduz a resposta de Dendroctonus frontalis (Scolytidae) para seu próprio feromônio quando simultaneamente liberado com o atrativo natural no campo (HAYES et al., 1994).

Os voláteis das folhas verdes de plantas não hospedeiras também têm sido mostrados inibir a resposta do feromônio de vários besouros-da-casca (POLAND; HAACK, 2000; HUBER; BORDEN, 2001). A atividade dos voláteis de plantas não hospedeiras poderia atuar como sinal negativo para os besouros-dacasca quando esses estão procurando árvores hospedeiras. Como foi discutido por Poland et al. (1998), poderia ser mais benéfico para esses coleópteros ter a capacidade de reconhecer e evitar um sinal volátil geral que é comumente emitido pelas árvores não hospedeiras do que reconhecer precisamente os voláteis espécie-específicos de cada espécie não hospedeira. Dessa maneira, várias espécies de árvores não hospedeiras com misturas de compostos voláteis coincidindo parcialmente poderiam ser percebidas e evitadas durante o processo de seleção do hospedeiro (POLAND et al., 1998). A atividade desses voláteis poderia atuar como um sinal negativo para os coleópteros quando estão à procura das coníferas durante o vôo, deixando de atacá-las. Os compostos voláteis de espécies não hospedeiras têm um grande potencial para a proteção das florestas contra os besouros-da-casca (ZHANG et al., 1999). Um outro exemplo é o Tomicus destruens (Coleoptera: Scolytidae), uma importante praga de pinheiros distribuída por toda a Europa. O benzil álcool, um semioquímico presente em extratos de Eucalyptus radiata (Myrtaceae), mas completamente ausente nos voláteis de folhas e caules dos pinheiros, induziu os besouros a abrirem um número limitado de galerias quando o composto químico foi depositado nos pinheiros cortados (GUERRERO et al., 1997). Esses resultados podem ter implicações importantes para o controle de T. destruens pela total exclusão desses das potenciais árvores hospedeiras ou regulação da densidade do ataque para níveis mais baixos de colonização da árvore.

\section{Influência da herbivoria nos voláteis liberados pelas plantas}

Os feromônios produzidos pelos insetos herbívoros podem fornecer sinais químicos aos predadores e parasitóides, orientando-os na localização de hospedeiros apropriados. Similarmente, muitos parasitóides e predadores se orientam pelo odor da planta hospedeira, inclusive pelos sinais químicos específicos liberados após a alimentação dos insetos herbívoros (THALER, 1999). Esses compostos, que incluem monoterpenos, sesquiterpenos, homoterpenos, compostos aromáticos e voláteis de folhas verdes, servem como um mecanismo de defesa da planta pela atração de predadores e parasitóides para o ataque das espécies pragas, deste modo reduzindo adicionais danos à planta. Foi verificado que os ésteres (Z)-3hexenil que são emitidos pelo tabaco após o dano de insetos influenciavam as fêmeas de Heliothis virescens (Lepidoptera: Noctuidae) a colocarem os ovos nas plantas danificadas, e alguns voláteis das folhas do repolho atraiam os parasitóides Trichogramma chilonis (Hymenoptera: Trichogrammatidae) e Cotesia plutellae (Hymenoptera: Braconidae) e o predador Chrysoperla carnea (Neuroptera: Chrysopidae), para P.xylostella, uma importante praga do repolho, de maneira similar ao efeito do feromônio sexual (DE MORAES et al., 2001, LIU; JIANG, 2003). As principais plantas hospedeiras dos besouros-da-casca Ips pini e I. grandicolis são o Pinus resinosa, Pinus banksiana e Pinus strobus, que contêm monoterpenos como seu volátil fitoquímico principal. Embora os monoterpenos sozinhos não atraiam predadores, alguns deles têm afetado significativamente a atração do predador para os feromônios de agregação.

Em muitos casos, os compostos voláteis emitidos pelas folhas como resultado do dano causado pelo inseto permitem aos parasitóides e predadores distinguir entre uma planta infestada ou não, e, portanto, ajuda-os a localizar hospedeiros ou presas (AGRAWAL, 2000). Esse é o caso das plantas de Phaseolus lunatus (Fabaceae), que produzem voláteis que atraem ácaros predadores quando danificadas por Tetranychus urticae (Acari: Tetranychidae) (TAKABAYASHI; DICKE, 1996). As plantas de algodão e 
milho liberam substâncias quando danificadas, pelas quais os himenópteros parasitóides são atraídos para atacar as lagartas de várias espécies de Lepidoptera (TUMLINSON et al., 1993).

A habilidade dos insetos para reconhecer e responder aos sinais químicos na localização do hospedeiro e distingui-los de outros odores indica que os voláteis emitidos pela planta devido ao dano de herbivoria são claramente distintos daqueles liberados em resposta a outros danos ou das plantas não atacadas. A planta tem a capacidade de diferenciar entre o dano provocado por um herbívoro ou outro dano e isto sugere uma reação específica associada com a alimentação do inseto (PARÉ; TUMLINSON, 1999). Em muitas plantas (algodão, tabaco), essa reação induz a emissão de altas concentrações de voláteis que são sintetizados em resposta à alimentação do inseto e não somente pelo dano mecânico. Somente duas secreções orais produzidas pela mastigação do inseto foram identificadas. A â-glucosidase presente nas lagartas de Pieris brassicae (Lepidoptera: Pieridae) e a glutamina presente nas lagartas de Spodoptera exígüa (Lepidoptera: Noctuidae) (MATTIACCI et al., 2005, ALBORN et al., 1997). Essas proteínas atuam com a mastigação do inseto e as plantas hospedeiras reagem a elas emitindo uma mistura de voláteis. Esses voláteis atraem as vespas parasitóides das lagartas, Cotesia glomerata e Cotesia marginiventris (Hymenoptera: Braconidae), que são inimigos naturais desses insetos, servindo como um mecanismo de defesa para a planta aos danos da herbivoria.

Embora a alimentação larval seja conhecida por provocar a emissão de voláteis em muitas plantas, a influência da oviposição dos insetos na emissão de voláteis só foi mostrada recentemente como um mecanismo de defesa indireto. Em plantas do gênero Ulmus (Ulmaceae), a oviposição de Xanthogaleruca luteola (Coleoptera: Chrysomelidae) induz a liberação de voláteis que são atrativos para o parasitóide de ovos Oomyzus gallerucae (Hymenoptera: Eulophidae) (MEINERS; HILKER, 2000). Nenhum dano artificial ou dano de alimentação nas folhas de Ulmus spp. induziu a produção de voláteis que atraem o parasitóide. A natureza da reação das plantas não foi identificada, mas o ácido jasmônico, um conhecido mediador de respostas das plantas pela alimentação de insetos herbívoros, tem sido demonstrado, por meio de estudos, mediar a produção de químicos que atraem parasitóides de ovos (MEINERS; HILKER, 2000).

A planta de tabaco Nicotiana attenuata (Solanaceae) produz uma enorme quantidade de nicotina em resposta ao ataque herbívoro, particularmente de Manduca sexta (Lepidoptera: Sphingidae). A nicotina é produzida e distribuída por toda a planta para a proteção (BALDWIN et al., 2002). Ela é conhecida como um dos melhores metabólicos de defesa da planta porque afeta os receptores de acetilcolina e desta maneira é tóxica para a maioria dos organismos heterotróficos com junções neuromusculares. Quando atacada por um inseto tolerante à nicotina, a planta reconhece o ataque pela rápida propagação de ácido jasmônico a partir do local do dano da herbivoria (SCHITTKO et al., 2000). O tratamento com ácido jasmônico aumenta as defesas diretas, incluindo a produção de toxinas (nicotina, fenólicos, flavonóides), proteínas antidigestivas (inibidoras de proteinase) e enzimas antinutritivas (polifenol oxidase), e, em adição, a planta emite uma extensa gama de voláteis como defesa indireta (BALDWIN et al., 2002).

\section{CONSIDERAÇÕES FINAIS}

O comportamento de insetos herbívoros é freqüentemente integrado com a planta hospedeira de diversas maneiras. Essa integração pode ser observada nos efeitos induzidos pelas plantas hospedeiras na fisiologia e comportamento dos insetos, inclusive na reprodução, e pelas respostas de defesa das plantas ao ataque de insetos.

Particularmente importantes são os efeitos das plantas hospedeiras em relação ao comportamento pelo feromônio, que parece ser parte das estratégias dos machos (maximizar encontros com as fêmeas) bem como das fêmeas (acesso a novos recursos de alimentação e locais de oviposição). O aumento da atração de machos, ou fêmeas, pelos odores da planta hospedeira sugere que possam ser desenvolvidas armadilhas mais efetivas para o controle de pragas. Iscas baseadas somente em feromônio sintético não são completamente atrativas para a maioria dos insetos e são improváveis de serem completamente competitivas com os sinais que emanam das plantas hospedeiras e dos alimentos. Baseada nesta estratégia, merece destaque o desenvolvimento de novas iscas para diversas espécies de insetos pragas. 
Os semioquímicos, naturalmente, são substâncias muito pouco tóxicas e atuam apenas sobre o comportamento dos organismos. Dentre os semioquímicos, os feromônios já são utilizados e comercializados mundialmente para o monitoramento, coleta massal e confundimento (interrupção de acasalamento) dos insetos. Outros semioquímicos, como os cairomônios e sinomônios, deverão ser comercializados a partir dos próximos anos, com o desenvolvimento e aprimoramento de novas técnicas de emprego nos diferentes ecossistemas.

Pode-se utilizar os semioquímicos para minimizar os custos de controle de pragas, por exemplo, combinando pequenas quantidades de ingredientes ativos feromonais, de custo elevado, acrescentando pequenas quantias de voláteis de plantas, mais baratos, misturados ao feromônio.

O sinergismo entre feromônios de insetos e os odores da planta hospedeira podem aumentar a atração de inimigos naturais, oferecendo novas estratégias para o controle biológico.

Novas pesquisas na área de ecologia química poderão esclarecer estas e outras questões relevantes para o desenvolvimento de programas de controle de pragas mais efetivos e seguros.

\section{REFERÊNCIAS}

AGRAWAL, A. A. Mechanisms, ecological consequences and agricultural implications of tri-trophic interactions. Current Opinion in Plant Biology, v. 3, p. 329-335, 2000.

ALBORN, H. T. et al. An elicitor of plant volatiles from beet armyworm oral secretion. Science, v. 276, p. 945-949, 1997.

BALDWIN, I. T. et al. Merging molecular and ecological approaches in plant-insect interactions. Current Opinion in Plant Biology, v. 4, p. 351-358, 2002.

BARKAWI, L. S. et al. Frontalin: de novo biosynthesis of an aggregation pheromone component by Dendroctonus spp. bark beetles (Coleoptera: Scolytidae). Insect Biochemistry and Molecular Biology, v. 33, p. 773-788, 2003.

BIRCH, M. C. Aggregation in bark beetle. In: BELL, W. J.; CARDÉ, R. T. (Ed.). Chemical ecology of insects. London: Chapman \& Hall, 1994. p. 319-321.

BOPPRE, M. Chemical communication, plant relationships, and mimicry in the evolution of danaid butterflies. Entomologia Experimentalis et Applicata, v. 24, p. 64-77, 1978.

BORDEN, J. H. Strategies and tactis for the use of semiochemicals against forest insect pests in North America. In: LUMSDEN, R. D.; VAUGHN, J. L. (Ed.). Pest management: Biologically based technologies. Washington, DC: Am. Chem. Soc., 2003. p. 265-279.

BYERS, J. A. Male specific conversion of the host plant compound myrcene to the pheromone (+)-ipsdienol in the bark beetle Dendroctonus brevicomis. Journal of Chemical Ecology, v. 82, p. 363-372, 1982.

CONNER, W. F. et al. Courtship pheromone production and body size as correlates of larval diet in males of the arctiid moth, Utethisa ornatrix. Journal of Chemical Ecology, v. 16, p. 543-552, 1990.

DE MORAES, C. M. et al. Caterpillar-induced nocturnal plant volatiles repel non-specific females. Nature, v. 410, p. 577-580, 2001.

DICKENS, J. C. Green leaf volatiles enhance aggregation pheromone of the boll weevil, Anthonomus grandis. Entomologia Experimentalis et Apllicata, v. 52, p. 191-203, 1989.

DRESSLER, R. L. Biology of the orchid bees (Euglossini). Annual Review of Ecology Systematics, v.13, p. 373-394, 1982.

DUSSOURD, D. E. et al. Biparental defense endowment of eggs with acquired plant alkaloid in a moth (Utetheisa ornatrix). Proceedings... National Academy of Sciences USA, v. 85, p. 992-996, 1988. 
EISNER, T.; MEINWALD, J. Alkaloid derived pheromones and sexual selection in Lepidoptera. In: BlOmQuist, G. J. (ed.). Pheromone biochemistry. New York: Academic, 1997. p. 251-269.

GONZÁLES, A.; ROSSINI, C.; EISNER, M. Sexually transmitted chemical defense in a moth Utetheisa ornatrix. Proceedings ... National Academy of Sciences USA, v. 96, p. 5570-5574, 1999.

GUERRERO, A. et al. Semiochemically induced inhibition of behaviour of Tomicus destruens (Woll.) (Coleoptera: Scolytidae). Naturwissenschaften, v. 84, p. 155-157, 1997.

HARTMAnN, T. Chemical ecology of pyrrolizidine alkaloids. Planta, v. 207, p. 483-495, 1999.

HARTMANN, T.; OBER, D. Biosynthesis and metabolism of pyrrolizidine alkaloids in plants and specialized insect herbivores. Topics in Current Chemistry, v. 209, p. 207-243, 2000.

HAYES, J. L. et al. Repellent properties of the host compound 4-allylanisole to the southern pine beetle. Journal of Chemical Ecology, v. 20, p. 1595-1615, 1994.

HENDRIKSE, A.; VON-BUNNEMYER, E. Role of host plant stimuli in sexual behavior of small ermine moths (Yponomeuta). Ecological Entomology, v. 12, p. 363-371, 1987.

HENDRY, L. B. Insect pheromones: diet related? Science, v. 192, p. 143-145, 1976.

HENDRY, L. B.; WICHMANN, J. K.; HINDENLANG, D. M. Evidence for origin of insect sex pheromones: presence in food plants. Science, v. 188, p. 59-62, 1975.

HUBER, D. P. W.; BORDEN, J. H. Angiosperm bark volatiles disrupt response of Douglas-fir beetle, Dendroctonus pseudotsugae, to attractant-baited traps. Journal of Chemical Ecology, v. 27, p. 217-233, 2001.

HUGHES, P. R. Effect of alpha-pinene exposure on trans-verbenol synthesis in Dendroctonus ponderosae Hopkins. Naturwissenschaften, v. 60, p. 261-262, 1973.

HUNT, D. W. A. et al. Sex-specific production of ipsdienol and myrcenol by Dendroctonus ponderosae (Coleoptera: Scolytidae) exposed to myrcene vapors. Journal of Chemical Ecology, v. 12, p. 1579-1586, 1986.

IYENGAR, V. K.; ROSSINI, C.; EISNER, T. Precopulatory assessment of male quality in na arctiid moth (Utetheisa ornatrix): hydroxydanaidal is the only criterion of choice. Behavior, Ecology and Sociobiology, v. 49, p. 283-288, 2001.

JAFFE, K. et al. Chemical ecology of the palm weevil Rhynchophorus palmarum (L.) (Coleoptera: Curculionidae): attraction to host plants and to a male-produced aggregation pheromone. Journal of Chemical Ecology, v. 19, p. 1703-1720, 1993.

LANDOLT, P. J., PHILLIPS, T. W. Host plant influences on sex pheromone behavior of phytophagous insects. Annual Review of Entomology, v. 42, p. 371-391, 1997.

LIU, S. S.; JIANG, L. H. Differential parasitism of Plutella xylostella (Lepidoptera: Plutellidae) larvae by the parasitoid Cotesia plutellae (Hymenoptera: Braconidae) on two host plant species. Bulletin of Entomological Research, v. 93, p. 65-72, 2003.

MATTIACI, L. et al. Beta-Glucosidase: an elicitor of herbivore induced plant odor that attracts host-searching parasitic wasps. Proceedings of the North Central Branch of the Entomological Society of America, v. 92, p. 2036-2040, 2005.

MCNEIL, J. N.; DELISLE, J. Are host plants important in pheromone mediated mating systems of Lepidoptera? Experientia, v. 45, p. 236-240, 1989.

MEINERS, T.; HILKER, M. Induction of plant synomones by oviposition of a phytophagous insect. Journal of Chemical Ecology, v. 26, p. 221-232, 2000. 
NASCIMENTO, R. R.; SANT'ANA, A. E. G. Feromônios de insetos: Biologia, química e emprego no manejo de pragas. 2. ed. Ribeirão Preto: Holos, p. 65-71, 2001.

NISHIDA, R. Sequestraion of defensive substances from plants by Lepidoptera. Annual Review of Entomology, v. 47, p. 57-92, 2002.

PARÉ, P. W.; TUMLINSON, J.H. Plant volatiles as a defense against insect herbivores. Plant Physiology, v. 121, p. 325-331, 1999.

POLAND, T. M. et al. Green leaf volatiles disrupt responses by the spruce beetle, Dendroctonus rufipennis, and the western pine beetle, Dendroctonus brevicomis (Coleoptera: Scolytidae) to attractant-baited traps. Journal of the Entomological Society of British Columbia, v. 95, p. 17-24, 1998.

POLAND, T. M.; HAACK, R. A. Pine shoot beetle, Tomicuspiniperda (Col., Scolytidae), responses to common green leaf volatiles. Journal of Applied Entomology, v. 124, p. 63-69, 2000.

RAFFA, K. F.; PHILLIPS, T. W.; SALOM, S. M. Strategies and mechanisms of host colonization by bark beetles. In: SHOWALTER, R. D.; FILIP, G. M. (Ed.). Beetle-patogen interactions in conifer forests. New York: Academic, 1996. p. 103-127.

RAINA, A. K. et al. Chemical signals from host plant and sexual behavior in a moth. Science, v. 255, p. $592-$ 594, 1992.

RAINA, A. K. Selected factors influencing neurohormonal regulation of sex pheromone production in Heliothis species. Journal of Chemical Ecology, v. 14, p. 2063-2069, 1988.

REDDY, G. V. P.; GUERRERO, A. Interactions of insects pheromones and plant semiochemicals. Trends in Plant Science, v. 9, p. 253-261, 2004.

RIDDIFORD, L. M.; WILLIAMS, C. M. Chemical signaling between polyphemus moths and between moths and host plant. Science, v. 156, p. 541, 1997.

ROCHAT, D. et al. Identification of pheromone synergists in American weevil, Rhynchophorus palmarus, and attraction of related Dynamis borassi. Journal of Chemical Ecology, v. 26, p. 155-187, 2000.

SCHITTKO, U. et al. Eating the evidence? Manduca sexta can not disrupt specific jasmonate induction in Nicotiana attenuata by rapid consumption. Planta, v. 210, p. 343-346, 2000.

SCHNEIDER, D. et al. A pheromone precursor and its uptake in male Danaus butterflies. Journal of Comparative Physiology, v. 97, p. 245-256, 1975.

SCHNEIDER, D. et al. Scent organ development in Creatonotos moths: regulation by pyrrolizidine alkaloids. Science, v. 215, p. 1264-1265, 1982.

TAKABAYASHI, J.; DICKE, M. Plant-carnivore mutualism through herbivore-induced carnivore attractants. Trends in Plant Science, v. 1, p. 109-113, 1996.

TEGONI, M.; CAMPANACCI, V.; CAMBILlAU, C. Structural aspects of sexual attraction and chemical communication in insects. Trends in Biochemical Sciences, v. 29, p. 257-264, 2004.

THALER, J. S. Jasmonate-inducible plant defenses cause increased parasitism of herbivores. Nature, v. 339 , p. 686-688, 1999.

TUMLINSON, J. H. et al. How parasitic wasps find their hosts. Scientific American, v. 268, p. 100-106, 1993.

WILLIAMS, N. H. The biology of orchids and euglossine bees. In: ARDITTI, J. (Ed.). Orchid biology: Reviews and perspectives. Ithaca, New York: Cornell Univ. Press, v. 80, p. 119-171, 2002.

WOOD, D. L. The role of pheromones, kairomones, and allomones in the host selection and colonization behavior of bark beetles. Annual Review of Entomology, v. 27, p. 411-446, 1982. 
ZHANG, Q; SCHLYTER, F.; ANDERSON, P. Green leaf volatiles interrupt pheromone response of spruce bark beetle, Ips typographus. Journal of Chemical Ecology, v. 25, p. 2847-2861, 1999.

Recebido: 22/05/2006

Aprovado: 30/06/2006 